CLINICAL STUDY

\title{
The clinical significance and primary determinants of hirsutism in patients with polycystic ovary syndrome
}

\author{
Dimitrios Panidis, Konstantinos Tziomalos ${ }^{1}$, Efstathios Papadakis, Panagiotis Chatzis, Eleni A Kandaraki, \\ Elena A Tsourdi, Christos Vosnakis and Ilias Katsikis \\ Division of Endocrinology and Human Reproduction, Second Department of Obstetrics and Gynecology, Hippokration Hospital, Aristotle University of \\ Thessaloniki, Thessaloniki, Greece and ${ }^{1}$ First Propedeutic Department of Internal Medicine, AHEPA Hospital, Aristotle University of Thessaloniki, \\ 1 Stilponos Kyriakidi Street, 54636 Thessaloniki, Greece \\ (Correspondence should be addressed to K Tziomalos; Email: ktziomalos@yahoo.com)
}

\begin{abstract}
Objective: Hirsutism is frequently present in patients with polycystic ovary syndrome (PCOS) and is a major sign of hyperandrogenism. However, other disorders frequently present in PCOS, particularly abdominal obesity and insulin resistance (IR), have also been implicated in the development of hirsutism in this population but relevant data are limited. We aimed to define the determinants of the presence of hirsutism in PCOS.

Design: Observational study.

Methods: We studied 1297 patients with PCOS (age $24.3 \pm 5.8$ years, BMI $26.8 \pm 6.9 \mathrm{~kg} / \mathrm{m}^{2}$ ). Hirsutism was defined as a modified Ferriman-Gallwey score $\geq 8$.

Results: Women with hirsutism were younger, had greater BMI, and had higher levels of circulating androgens than women without hirsutism; markers of IR did not differ between the two groups after adjustment for age and BMI. The prevalence of hirsutism progressively declined with age, was lower in normal-weight women than in overweight and obese women, and was comparably prevalent in the hyperandrogenemic phenotypes of PCOS. In binary logistic regression analysis, independent predictors of the presence of hirsutism were younger age, larger waist circumference (W), and higher serum testosterone levels. In stepwise linear regression analysis, the Ferriman-Gallwey score independently correlated with age, $\mathrm{W}$, free androgen index, and serum $\Delta_{4}$-androstenedione and DHEAS levels. Conclusions: Besides hyperandrogenemia, abdominal obesity, and young age are independently associated with the presence of hirsutism. In contrast, the relationship between IR and hirsutism appears to be mediated by the more severe obesity of insulin-resistant patients with PCOS.
\end{abstract}

European Journal of Endocrinology 168 871-877

\section{Introduction}

Androgen excess is a pivotal characteristic of polycystic ovary syndrome (PCOS) (1). It has been argued that PCOS is primarily a disorder of androgen excess and that hyperandrogenemia or clinical hyperandrogenism is sine qua non for the diagnosis of the syndrome (2). Even though this position is a matter of controversy, clinical or biochemical signs of hyperandrogenism are included in the current diagnostic criteria of PCOS (3).

Hirsutism is a major sign of hyperandrogenism and is defined as excessive terminal hair that appears in a male pattern in a woman (4). Androgens play a major role in the development of hirsutism (5). However, other disorders frequently present in patients with PCOS, particularly abdominal obesity and insulin resistance (IR), have also been implicated in the development of hirsutism in this population $(6,7,8$, 9, 10, 11). However, only a limited number of small studies have evaluated the characteristics of hirsute patients with PCOS and have yielded conflicting results $(6,7,8,9,10,11)$.

The determination of the endocrine and metabolic characteristics of hirsute patients with PCOS is an important aspect of the management of this disorder because hirsutism is highly prevalent in this population $(6,7,8,12,13)$, adversely affects health-related quality of life (14), and might play a role in the increased cardiovascular risk of these patients $(15,16)$. Accordingly, we aimed to define the characteristics of hirsute patients with PCOS in a large cohort of patients with this syndrome.

\section{Materials and methods}

\section{Subjects}

We studied 1297 women with PCOS (age $24.3 \pm 5.8$ years, BMI $26.8 \pm 6.9 \mathrm{~kg} / \mathrm{m}^{2}$ ). All of them were outpatients at the Gynecological Endocrinology Infirmary of the 
Second Department of Obstetrics and Gynecology, Aristotle University of Thessaloniki, Greece.

Diagnosis of PCOS was based on the revised criteria of Rotterdam, which require the presence of at least two of the following three features: i) oligo- or anovulation ( $<8$ spontaneous hemorrhagic episodes/year); ii) biochemical hyperandrogenemia (defined in our population as early follicular phase testosterone $>60 \mathrm{ng} / \mathrm{dl}$, corresponding to the mean +2 s.D. testosterone levels in 200 control subjects measured in our laboratory) or clinical manifestations of hyperandrogenemia (Ferriman-Gallwey score $\geq 8$ ); and iii) polycystic ovaries on ultrasound ( $\geq 12$ small follicles in at least one ovary and/or ovarian volume $>10 \mathrm{~cm}^{3}$ ) (3).

None of the women studied had galactorrhea or any endocrine or systemic disease that could possibly affect reproductive physiology. No woman reported use during the last semester of any medication that could interfere with the normal function of the hypothalamic-pituitary-gonadal axis. When basic $17 \alpha$-hydroxyprogesterone $(17 \alpha-\mathrm{OHP})$ levels were $>1.5 \mathrm{ng} / \mathrm{ml}$, the Synacthen test $(0.25 \mathrm{mg} / 1 \mathrm{ml}$; Novartis Pharma S.A., Rueil-Malmaison, France) was performed to rule out congenital adrenal hyperplasia. Other causes of hyperandrogenemia, including prolactinoma, Cushing's syndrome, and androgen-secreting tumors, were also excluded.

Informed consent was obtained from all women, and the study was approved by the Institutional Review Board. The study met the requirements of the 1975 Helsinki guidelines.

\section{Study protocol}

In all women, body weight, height, waist circumference $(\mathrm{W})$, and hip circumference $(\mathrm{H})$ were measured. Body weight was measured with analog scales and in light clothing; height was measured barefoot with a stadiometer. The BMI was calculated by dividing weight $(\mathrm{kg})$ by height squared $\left(\mathrm{m}^{2}\right)$ to assess obesity. The $\mathrm{W}$ was obtained as the smallest circumference at the level of the umbilicus and the $\mathrm{H}$ was measured at the level of the widest diameter around the buttocks. The $\mathrm{W} / \mathrm{H}$ was calculated by dividing $\mathrm{W}$ by $\mathrm{H}$.

Hirsutism was defined as a modified FerrimanGallwey score $\geq 8(17,18)$. None of the patients had received oral contraceptives or other antiandrogens in the past.

Baseline blood samples were collected between 3 and 7 days after a spontaneous bleeding episode, after an overnight fast. The circulating levels of FSH, $\mathrm{LH}$, prolactin (PRL), testosterone, $\Delta_{4}$-androstenedione $\left(\Delta_{4}-\mathrm{A}\right)$, DHEAS, $17 \alpha$-OHP, sex hormone-binding globulin (SHBG), glucose, and insulin were measured. Immediately after the baseline blood sampling, an oral glucose tolerance test was performed; 75 g glucose were administered orally and serum glucose levels were determined after 30, 60, 90, and $120 \mathrm{~min}$. On the same day, transvaginal ultrasonography was performed and the volume of each ovary was determined, as well as the number of follicles in each ovary.

The study population was divided according to: i) age in $\leq 20$ years old $(n=381), 21-30$ years old $(n=717)$, and $>30$ years old $(n=199)$; ii) BMI in normal weight (i.e. with BMI $<25.0 \mathrm{~kg} / \mathrm{m}^{2} ; n=679$ ), overweight (i.e. with BMI $25.0-29.9 \mathrm{~kg} / \mathrm{m}^{2} ; n=277$ ), and obese (i.e. with BMI $\geq 30.0 \mathrm{~kg} / \mathrm{m}^{2} ; n=341$ ); iii) PCOS phenotype in women with phenotype 1 (i.e. with oligo- or anovulation, hyperandrogenism, and polycystic ovaries; $n=653$ ), phenotype 2 (i.e. with oligo- or anovulation and hyperandrogenism but without polycystic ovaries; $n=408$ ), phenotype 3 (i.e. with hyperandrogenism and polycystic ovaries but without oligo- or anovulation; $n=131$ ), and phenotype 4 (i.e. with oligo- or anovulation and polycystic ovaries but without hyperandrogenism; $n=105$ ) (Table 1) (3); and iv) hyperandrogenism in women with clinical but not biochemical hyperandrogenism $(n=136)$ and women with both clinical and biochemical hyperandrogenism $(n=644)$.

\section{Methods}

Serum glucose, insulin, FSH, LH, PRL, androgens, and $17 \alpha$-OHP concentrations were measured as described previously (19). Free androgen index (FAI) was determined as follows: $\mathrm{FAI}=$ testosterone $(\mathrm{nmol} / \mathrm{l}) \times$ 100/SHBG (nmol/l) (20). The homeostasis model assessment of IR (HOMA-IR) index was calculated as follows: HOMA-IR = fasting insulin $(\mathrm{mIU} / \mathrm{l}) \times$ glucose $(\mathrm{mg} / \mathrm{dl}) / 405(21)$. The quantitative insulin sensitivity check index (QUICKI) was calculated according to the following formula: $\mathrm{QUICKI}=1 /(\log$ insulin $(\mathrm{mIU} / \mathrm{l})+\log$ glucose $(\mathrm{mg} / \mathrm{dl}))(22)$.

Table 1 Definition of the phenotypes of the polycystic ovary syndrome (PCOS).

\begin{tabular}{|c|c|c|c|}
\hline $\begin{array}{l}\text { PCOS } \\
\text { phenotype }\end{array}$ & Anovulation & $\begin{array}{l}\text { Biochemical hyperandrogenemia } \\
\text { or clinical manifestations of } \\
\text { hyperandrogenemia }\end{array}$ & $\begin{array}{l}\text { Polycystic ovaries } \\
\text { in transvaginal } \\
\text { ultrasonography }\end{array}$ \\
\hline 1 (severe PCOS) & + & + & + \\
\hline $\begin{array}{l}2 \text { (anovulation and } \\
\text { hyperandrogenemia) }\end{array}$ & + & + & - \\
\hline 3 (ovulatory PCOS) & - & + & + \\
\hline 4 (mild PCOS) & + & - & + \\
\hline
\end{tabular}


Table 2 Characteristics of women with polycystic ovary syndrome (PCOS) and hirsutism and of women with PCOS without hirsutism.

\begin{tabular}{|c|c|c|c|}
\hline & $\begin{array}{c}\text { Women with } \\
\text { hirsutism }(n=780)\end{array}$ & $\begin{array}{l}\text { Women without } \\
\text { hirsutism }(n=517)\end{array}$ & $\begin{array}{l}\boldsymbol{P} \text { (adjusted for } \\
\text { age and BMI) }\end{array}$ \\
\hline Age (years) & $23.8 \pm 5.6$ & $25.1 \pm 5.9$ & $<0.001$ \\
\hline BMI $\left(\mathrm{kg} / \mathrm{m}^{2}\right)$ & $27.3 \pm 7.0$ & $25.9 \pm 6.8$ & $<0.001$ \\
\hline Waist $(\mathrm{cm})$ & $84.9 \pm 15.6$ & $81.9 \pm 15.0$ & NS \\
\hline W/H & $0.79 \pm 0.07$ & $0.79 \pm 0.29$ & NS \\
\hline Ferriman-Gallwey score & $11.4 \pm 2.5$ & $3.4 \pm 2.4$ & $<0.001$ \\
\hline FSH (mlU/ml) & $5.8 \pm 1.7$ & $6.0 \pm 1.9$ & NS \\
\hline LH (mlU/ml) & $7.8 \pm 5.9$ & $7.7 \pm 4.9$ & NS \\
\hline Prolactin $(\mathrm{ng} / \mathrm{ml})$ & $14.4 \pm 7.3$ & $13.6 \pm 7.1$ & NS \\
\hline Testosterone (ng/dl) & $78.4 \pm 32.5$ & $68.6 \pm 26.9$ & $<0.001$ \\
\hline$\Delta_{4}-\mathrm{A}(\mathrm{ng} / \mathrm{ml})$ & $2.9 \pm 1.2$ & $2.7 \pm 1.0$ & 0.001 \\
\hline DHEAS (ng/ml) & $3057.4 \pm 1283.6$ & $2755.9 \pm 1246.3$ & 0.001 \\
\hline FAI & $9.92 \pm 7.99$ & $6.75 \pm 5.04$ & $<0.001$ \\
\hline $17 \alpha-\mathrm{OHP}(\mathrm{ng} / \mathrm{ml})$ & $1.1 \pm 0.5$ & $1.1 \pm 0.6$ & NS \\
\hline $\mathrm{SHBG}(\mathrm{nmol} / \mathrm{ml})$ & $38.0 \pm 23.8$ & $47.9 \pm 27.3$ & $<0.001$ \\
\hline Glucose (mg/dl) & $97.8 \pm 33.3$ & $97.5 \pm 12.1$ & NS \\
\hline Insulin $(\mu \mathrm{lU} / \mathrm{ml})$ & $13.5 \pm 13.8$ & $11.7 \pm 8.9$ & NS \\
\hline Glucose/insulin & $11.01 \pm 7.29$ & $12.48 \pm 8.69$ & NS \\
\hline AUC OGTT & $15491.9 \pm 3200.4$ & $15161.3 \pm 3319.6$ & NS \\
\hline HOMA-IR & $3.35 \pm 3.80$ & $2.89 \pm 2.51$ & NS \\
\hline QUICKI & $0.33 \pm 0.03$ & $0.34 \pm 0.03$ & NS \\
\hline Ovarian volume $\left(\mathrm{cm}^{3}\right)$ & $7.9 \pm 3.5$ & $8.6 \pm 4.7$ & 0.006 \\
\hline Ovarian follicles & $11.0 \pm 4.8$ & $12.0 \pm 6.6$ & $<0.001$ \\
\hline
\end{tabular}

NS, not significant; W/H, waist:hip ratio; $\Delta_{4}-\mathrm{A}, \Delta_{4}$-androstenedione; FAI, free androgen index; $17 \alpha-\mathrm{OHP}$, $17 \alpha$-hydroxyprogesterone; SHBG, sex hormone-binding globulin; AUC OGTT, area under the curve of the oral glucose tolerance test; HOMA-IR, homeostasis model assessment of insulin resistance; QUICKI, quantitative insulin sensitivity check index.

\section{Transvaginal ultrasonography}

Transvaginal ultrasonography was performed by an experienced operator in all women. Ovarian volume was calculated as follows: ovarian volume $=(\pi / 6)$ $\times$ ovarian length $\times$ ovarian height $\times$ ovarian width. The presence of polycystic ovaries was diagnosed by the presence of 12 or more follicles in each ovary measuring $2-9 \mathrm{~mm}$ in diameter and/or increased ovarian volume $\left(>10 \mathrm{~cm}^{3}\right)$.

\section{Statistical analysis}

Data analysis was performed with the statistical package SPSS (version 17.0; SPSS, Inc., Chicago, IL, USA). Data are reported as mean \pm s.D. Differences in continuous variables between groups were assessed with analysis of covariance adjusting for age and BMI. Differences in the prevalence of hirsutism between the age and BMI groups and the PCOS phenotypes were assessed with the $\chi^{2}$ test. Binary logistic regression analysis was used to identify independent predictors of the presence of hirsutism.

Correlations between the Ferriman-Gallwey score and other parameters were assessed with Pearson's correlation. Parameters that were significantly correlated with the Ferriman-Gallwey score according to Pearson's correlation were included in a stepwise linear regression analysis model to identify parameters that were independently correlated with the FerrimanGallwey score. In all cases, a $P$ value $<0.05$ was considered significant.

\section{Results}

The mean Ferriman-Gallwey score was $8.2 \pm 4.6$ and hirsutism was present in $60.1 \%$ of the study population. Characteristics of women with hirsutism and of those without hirsutism are shown in Table 2. Women with hirsutism were younger and had greater BMI ( $P<0.001$ for both comparisons). In age and BMIadjusted comparisons, women with hirsutism had higher levels of circulating androgens (serum testosterone, $\Delta_{4}-\mathrm{A}$, and DHEAS levels and FAI) than women without hirsutism. In contrast, markers of IR did not differ between women with hirsutism and those without hirsutism.

Anthropometric parameters and markers of IR in women with clinical but not biochemical hyperandrogenism and in women with both clinical and biochemical hyperandrogenism are shown in Table 3 . Women with clinical but not biochemical hyperandrogenism were older and had smaller BMI and W than women with both clinical and biochemical hyperandrogenism $(P<0.001, P<0.001$, and $P=0.038$ respectively $)$. In age- and BMI-adjusted comparisons, none of the markers of IR differed between these two groups. 
Table 3 Anthropometric parameters and markers of insulin resistance in women with clinical but not biochemical hyperandrogenism and in women with both clinical and biochemical hyperandrogenism.

\begin{tabular}{lccc}
\hline & $\begin{array}{c}\text { Women with clinical } \\
\text { but not biochemical } \\
\text { hyperandrogenism }(n=136)\end{array}$ & $\begin{array}{c}\text { Women with both clinical } \\
\text { and biochemical } \\
\text { hyperandrogenism }(n=644)\end{array}$ & $\begin{array}{c}\boldsymbol{P} \text { (adjusted for } \\
\text { age and BMI) }\end{array}$ \\
\hline Age (years) & $25.7 \pm 6.5$ & $23.4 \pm 5.3$ & $<0.001$ \\
BMl (kg/m $\left.{ }^{2}\right)$ & $25.2 \pm 6.7$ & $27.8 \pm 7.0$ & $<0.001$ \\
Waist (cm) & $80.3 \pm 14.4$ & $85.9 \pm 17.4$ & 0.038 \\
W/H & $0.77 \pm 0.07$ & $0.79 \pm 0.08$ & $\mathrm{NS}$ \\
Ferriman-Gallwey score & $10.6 \pm 2.1$ & $11.5 \pm 2.6$ & 0.009 \\
Glucose (mg/dl) & $97.4 \pm 11.7$ & $97.8 \pm 36.2$ & $\mathrm{NS}$ \\
Insulin $(\mu / \mathrm{m} / \mathrm{ml})$ & $11.0 \pm 9.9$ & $14.1 \pm 14.4$ & $\mathrm{NS}$ \\
Glucose/insulin & $13.42 \pm 8.66$ & $10.50 \pm 6.88$ & $\mathrm{NS}$ \\
AUC OGTT & $15205.5 \pm 2775.3$ & $15561.7 \pm 3294.5$ & $\mathrm{NS}$ \\
HOMA-IR & $2.69 \pm 2.51$ & $3.49 \pm 4.01$ & $\mathrm{NS}$ \\
QUICKI & $0.34 \pm 0.03$ & $0.33 \pm 0.03$ & $\mathrm{NS}$ \\
\hline
\end{tabular}

NS, not significant; W/H, waist:hip ratio; AUC OGTT, area under the curve of the oral glucose tolerance test; HOMA-IR, homeostasis model assessment of insulin resistance; QUICKI, quantitative insulin sensitivity check index.

The prevalence of hirsutism progressively declined with age, being $65.6,60.1$, and $50.0 \%$ in women $\leq 20$, $21-30$, and $>30$ years old respectively $(P=0.001)$. In addition, the prevalence of hirsutism was similar in overweight and obese women (67.1 and 65.6\% respectively) but was significantly lower in normalweight women $(54.6 \% ; P<0.001$, compared with overweight and obese women). Finally, hirsutism was comparably prevalent in phenotypes 1,2 , and 3 (64.6, 67.6 , and $62.6 \%$ respectively). By definition, none of the patients with phenotype 4 had hirsutism $(P<0.001$ compared with the other phenotypes). The FerrimanGallway score did not differ significantly between phenotypes 1,2 , and $3(8.7 \pm 4.4,8.6 \pm 4.5$, and $8.4 \pm 4.7$ respectively) but was significantly lower in patients with phenotype $4(3.1 \pm 2.4, P<0.001$, vs all other phenotypes). In binary logistic regression analysis, independent predictors of the presence of hirsutism were age, $\mathrm{W}$, and serum testosterone levels $(P<0.001$ for all parameters; Table 4$)$.

Correlations between the Ferriman-Gallwey score and other parameters in univariate analysis in the total study population $(n=1.297)$ are shown in Table 5 . In stepwise linear regression analysis, the FerrimanGallwey score independently correlated with age, W, FAI, and serum $\Delta_{4}$-A and DHEAS levels $(P<0.001$, $P=0.002, \quad P=0.001, P=0.019$, and $P=0.022$, respectively; Table 6). In the subpopulation of women with clinical but not biochemical hyperandrogenism $(n=136)$, in univariate analysis, the Ferriman-Gallwey score correlated only with the FAI $(r=0.187, P=0.029)$.

\section{Discussion}

Patients with hirsutism had higher serum testosterone, $\Delta_{4}$-A and DHEAS levels, and FAI than patients without hirsutism. Moreover, the Ferriman-Gallwey score independently correlated with serum $\Delta_{4}$-A and DHEAS levels and the FAI. These findings are in agreement with previous reports $(6,7,11)$ and are somewhat expected given that hirsutism is a major clinical sign of hyperandrogenism $(5,18)$. Notably, only two small studies in patients with PCOS $(n=24$ and 58 respectively) did not identify significant differences in circulating androgens between patients with hirsutism and those without hirsutism, but this was probably due to the small sample size $(8,23)$. On the other hand, we should emphasize that circulating androgens were measured by an immunoassay method that has poor specificity and accuracy for androgens at low circulating concentrations (24). This represents a limitation of our study.

Patients with hirsutism were more obese in our study and the prevalence of hirsutism was lower in normalweight women than in overweight/obese women. Moreover, $\mathrm{W}$ was an independent predictor of the presence of hirsutism and correlated with the Ferriman-Gallwey score independently from circulating androgens. Previous studies also reported an association between obesity and more severe hirsutism $(6,7)$. In addition, lifestyle changes aiming at weight loss also reduced the Ferriman-Gallwey score (25). However, in other studies in women with PCOS, the FerrimanGallwey score did not correlate with WHR (8) or with BMI (9). However, the former study included mostly normal-weight women (8) and the latter (9) did not assess the correlation between the Ferriman-Gallwey score and W or WHR, which appear to be more sensitive markers of abdominal obesity than BMI $(6,26)$.

Table 4 Independent predictors of the presence of hirsutism in women with polycystic ovary syndrome.

\begin{tabular}{lccc}
\hline Variable & Odds ratio & $95 \% \mathbf{C l}$ & $\boldsymbol{P}$ \\
\hline Age & 0.962 & $0.942-0.982$ & $<0.001$ \\
Waist & 1.008 & $1.004-1.012$ & $<0.001$ \\
Testosterone & 1.016 & $1.008-1.025$ & $<0.001$ \\
\hline
\end{tabular}


IR appears to aggravate hyperandrogenemia in patients with PCOS (1). In addition, insulin-sensitizing agents have a small beneficial effect on hirsutism in this population (27). However, markers of IR did not differ between patients with hirsutism and those without hirsutism in our study after adjustment for age and BMI. Moreover, none of the markers of IR predicted the presence of hirsutism nor correlated with the Ferriman-Gallwey score after adjusting for W and circulating androgens. These findings are in agreement with most previous studies, which did not detect differences in markers of IR between patients with hirsutism and those without hirsutism but with similar BMI $(8,10)$. In reports that showed more severe IR in patients with hirsutism, the former were also more obese than patients without hirsutism (11). However, in a smaller study in 130 patients with PCOS, those with hirsutism were more insulin resistant than those without hirsutism even though the BMI and W were similar in the two groups (6). Overall, most data do not suggest an independent association between hirsutism and IR in patients with PCOS but more studies are needed to evaluate this relationship.

We observed a progressive decline in the prevalence of hirsutism with age. Moreover, age was an independent predictor of the presence of hirsutism and independently correlated with the Ferriman-Gallwey score. We are not aware of other studies that assessed the relationship between age and hirsutism in patients with PCOS. The improvement in hirsutism with age might be due to the progressive decline in circulating androgens during reproductive age in women with PCOS $(28,29)$. Given the adverse effects of hirsutism on quality of life in patients with PCOS (14), informing hirsute women with PCOS about the gradual decline in the prevalence of hirsutism with aging might have beneficial effects on their psychosocial status.

Table 5 Correlations between the Ferriman-Gallwey score and other parameters in univariate analysis in the total study population $(n=1297)$.

\begin{tabular}{lrr}
\hline Parameter & $\boldsymbol{r}$ & $\boldsymbol{P}$ \\
\hline Positive correlations & & \\
BMI & 0.125 & $<0.001$ \\
Waist circumference & 0.117 & $<0.001$ \\
Testosterone & 0.221 & $<0.001$ \\
$\Delta_{4}$-Androstenedione & 0.144 & $<0.001$ \\
DHEAS & 0.170 & $<0.001$ \\
Free androgen index & 0.280 & $<0.001$ \\
17 $\alpha$-Hydroxyprogesterone & 0.061 & 0.027 \\
Insulin & 0.097 & $<0.001$ \\
Homeostasis model assessment & 0.080 & 0.004 \\
of insulin resistance & & $<0.001$ \\
Negative correlations & & 0.002 \\
Age & -0.132 & $<0.001$ \\
FSH & -0.085 & $<0.001$ \\
Sex hormone-binding globulin & -0.224 & $<0.001$ \\
Glucose:insulin ratio & -0.119 & \\
Quantitative insulin sensitivity & -0.106 & \\
check index & & \\
\hline
\end{tabular}

Table 6 Stepwise linear regression analysis of the correlation between the Ferriman-Gallwey score and other parameters.

\begin{tabular}{llc}
\hline Model & Predictors & $\boldsymbol{R}^{2}$ \\
\hline 1 & FAl & 0.55 \\
2 & FAl, age & 0.64 \\
3 & FAl, age, DHEAS & 0.69 \\
4 & FAl, age, DHEAS, W & 0.75 \\
5 & FAl, age, DHEAS, W, $\Delta_{4}$-A & 0.79 \\
\hline
\end{tabular}

$\mathrm{FAl}$, free androgen index; $\mathrm{W}$, waist circumference; $\Delta_{4}-\mathrm{A}, \Delta_{4}$-androstenedione.

In our study, the prevalence of hirsutism and the Ferriman-Gallwey score did not differ significantly between women with the different hyperandrogenemic phenotypes of PCOS (i.e. phenotypes 1, 2, and 3). This is in agreement with previous reports $(30,31)$, whereas other groups reported a higher Ferriman-Gallwey score in patients with phenotypes 1 or 2 than in patients with phenotype $3(32,33)$ and in patients with phenotype 1 than in patients with phenotype 2 (34). Hyperandrogenemia was more pronounced in phenotypes characterized by more severe hirsutism in the latter studies $(32,33,34)$, whereas circulating androgens did not differ between phenotypes 1, 2, and 3 in studies that did not identify differences in hirsutism between these phenotypes $(30,31)$. In contrast, in our study, despite the similar Ferriman-Gallwey score in phenotypes 1, 2, and 3, circulating androgens were higher in phenotypes 1 and 2 than in phenotype 3 and were also higher in phenotype 1 than in phenotype 3 (data not shown). Therefore, our findings suggest that the Ferriman-Gallwey score is not adequately sensitive to detect subtle differences in circulating androgens between the hyperandrogenemic phenotypes of PCOS.

In conclusion, besides hyperandrogenemia, abdominal obesity and young age are independently associated with the presence of hirsutism. In contrast, the relationship between IR and hirsutism appears to be mediated by the more severe obesity of insulin-resistant patients with PCOS. Given the beneficial effects of lifestyle changes on both circulating androgens and obesity (25), they should represent the first-line management of hirsutism in PCOS. In addition, as hirsutism appears to be associated with increased cardiovascular risk in patients with PCOS $(15,16)$, lifestyle changes might also prevent or delay the progression of atherosclerosis in this population.

\section{Declaration of interest}

The authors declare that there is no conflict of interest that could be perceived as prejudicing the impartiality of the research reported.

\section{Funding}

This research did not receive any specific grant from any funding agency in the public, commercial or not-for-profit sector. 


\section{References}

1 Goodarzi MO, Dumesic DA, Chazenbalk G \& Azziz R. Polycystic ovary syndrome: etiology, pathogenesis and diagnosis. Nature Reviews. Endocrinology 20117 219-231. (doi:10.1038/nrendo. 2010.217)

2 Azziz R, Carmina E, Dewailly D, Diamanti-Kandarakis E, Escobar-Morreale HF, Futterweit W, Janssen OE, Legro RS, Norman RJ, Taylor AE et al. Positions statement: criteria for defining polycystic ovary syndrome as a predominantly hyperandrogenic syndrome: an Androgen Excess Society guideline. Journal of Clinical Endocrinology and Metabolism 2006 $914237-4245$. (doi:10.1210/jc.2006-0178)

3 Rotterdam ESHRE/ASRM-Sponsored PCOS consensus workshop group. Revised 2003 consensus on diagnostic criteria and longterm health risks related to polycystic ovary syndrome (PCOS). Human Reproduction 200419 41-47. (doi:10.1093/humrep/ deh098)

4 Martin KA, Chang RJ, Ehrmann DA, Ibanez L, Lobo RA, Rosenfield RL, Shapiro J, Montori VM \& Swiglo BA. Evaluation and treatment of hirsutism in premenopausal women: an endocrine society clinical practice guideline. Journal of Clinical Endocrinology and Metabolism 200893 1105-1120. (doi:10.1210/ jc.2007-2437)

5 Randall VA. Androgens and hair growth. Dermatologic Therapy 200821 314-328. (doi:10.1111/j.1529-8019.2008.00214.x)

6 Amato MC, Galluzzo A, Merlino S, Mattina A, Richiusa P, Criscimanna A \& Giordano C. Lower insulin sensitivity differentiates hirsute from non-hirsute Sicilian women with polycystic ovary syndrome. European Journal of Endocrinology 2006155 859-865. (doi:10.1530/eje.1.02290)

7 Guzel AI, Kuyumcuoğlu U \& Celik Y. Factors affecting the degree of hirsutism in patients with polycystic ovary syndrome. Archives of Gynecology and Obstetrics 2012285 767-770. (doi:10.1007/ s00404-011-2077-5)

8 Cosar E, Koken G, Sahin FK, Arioz DT \& Yilmazer M. Insulin sensitivity does not differentiate by hirsutism in non-obese women with polycystic ovary syndrome. Endocrine Journal $2008 \mathbf{5 5}$ 465-468. (doi:10.1507/endocrj.K07-069)

9 Landay M, Huang A \& Azziz R. Degree of hyperinsulinemia, independent of androgen levels, is an important determinant of the severity of hirsutism in PCOS. Fertility and Sterility 200992 643-647. (doi:10.1016/j.fertnstert.2008.06.021)

10 Chang WY, Knochenhauer ES, Bartolucci AA \& Azziz R. Phenotypic spectrum of polycystic ovary syndrome: clinical and biochemical characterization of the three major clinical subgroups. Fertility and Sterility 200583 1717-1723. (doi:10.1016/ j.fertnstert.2005.01.096)

11 Falsetti L, Gambera A, Andrico S \& Sartori E. Acne and hirsutism in polycystic ovary syndrome: clinical, endocrine-metabolic and ultrasonographic differences. Gynecological Endocrinology 200216 275-284.

12 Demir B, Pasa S, Demir S, Tumer C, Atay AE, Gul T \& Atamer Y. Hirsutism score and the severity of hyperandrogenism associated with polycystic ovary syndrome in the southeastern region of Turkey. Journal of International Medical Research 201139 1529-1535. (doi:10.1177/147323001103900443)

13 Legro RS, Myers ER, Barnhart HX, Carson SA, Diamond MP, Carr BR, Schlaff WD, Coutifaris C, McGovern PG, Cataldo NA et al. The pregnancy in polycystic ovary syndrome study: baseline characteristics of the randomized cohort including racial effects. Fertility and Sterility 200686 914-933. (doi:10.1016/j.fertnstert. 2006.03.037)

14 Coffey S \& Mason H. The effect of polycystic ovary syndrome on health-related quality of life. Gynecological Endocrinology $2003 \mathbf{1 7}$ 379-386. (doi:10.1080/09513590312331290268)

15 Luque-Ramírez M, Mendieta-Azcona C, Alvarez-Blasco F \& Escobar-Morreale HF. Androgen excess is associated with the increased carotid intima-media thickness observed in young women with polycystic ovary syndrome. Human Reproduction 200722 3197-3203. (doi:10.1093/humrep/dem324)

16 Christakou CD \& Diamanti-Kandarakis E. Role of androgen excess on metabolic aberrations and cardiovascular risk in women with polycystic ovary syndrome. Women's Health 20084 583-594. (doi:10.2217/17455057.4.6.583)

17 Hatch R, Rosenfield RL, Kim MH \& Tredway D. Hirsutism: implications, etiology, and management. American Journal of Obstetrics and Gynecology 1981140 815-830.

18 Escobar-Morreale HF, Carmina E, Dewailly D, Gambineri A, Kelestimur F, Moghetti P, Pugeat M, Qiao J. Wijeyaratne CN, Witchel SF et al. Epidemiology, diagnosis and management of hirsutism: a consensus statement by the Androgen Excess and Polycystic Ovary Syndrome Society. Human Reproduction Update 201218 146-170. (doi:10.1093/humupd/dmrO42)

19 Piouka A, Farmakiotis D, Katsikis I, Macut D, Gerou S \& Panidis D. Anti-Müllerian hormone levels reflect severity of PCOS but are negatively influenced by obesity: relationship with increased luteinizing hormone levels. American Journal of Physiology. Endocrinology and Metabolism 2009296 E238-E243. (doi:10.1152/ ajpendo.90684.2008)

20 Carter GD, Holland SM, Alaghband-Zadeh J, Rayman G, DorringtonWard P \& Wise PH. Investigation of hirsutism: testosterone is not enough. Annals of Clinical Biochemistry 198320 262-263.

21 Matthews D, Hosker J, Rudenski A, Naylor BA, Treacher DF \& Turner RC. Homeostasis model assessment: insulin resistance and $\beta$-cell function from fasting plasma glucose and insulin concentrations in man. Diabetologia 198528 412-419. (doi:10.1007/ BF00280883)

22 Katz A, Nambi SS, Mather K, Baron AD, Follmann DA, Sullivan G \& Quon MJ. Quantitative insulin sensitivity check index: a simple, accurate method for assessing insulin sensitivity in humans. Journal of Clinical Endocrinology and Metabolism 200085 2402-2410. (doi:10.1210/jc.85.7.2402)

23 Lobo RA, Goebelsmann U \& Horton R. Evidence for the importance of peripheral tissue events in the development of hirsutism in polycystic ovary syndrome. Journal of Clinical Endocrinology and Metabolism 198357 393-397. (doi:10.1210/jcem-57-2-393)

24 Norman RJ, Dewailly D, Legro RS \& Hickey TE. Polycystic ovary syndrome. Lancet 2007 370 685-697. (doi:10.1016/ S0140-6736(07)61345-2)

25 Moran LJ, Hutchison SK, Norman RJ \& Teede HJ. Lifestyle changes in women with polycystic ovary syndrome. Cochrane Database of Systematic Reviews 20117 CD007506.

26 Yusuf S, Hawken S, Öunpuu S, Dans T, Avezum A, Lanas F, McQueen M, Budaj A, Pais P, Varigos J et al. Effect of potentially modifiable risk factors associated with myocardial infarction in 52 countries (the INTERHEART study): case-control study. Lancet 2004364 937-952. (doi:10.1016/S0140-6736(04)17018-9)

27 Cosma M, Swiglo BA, Flynn DN, Kurtz DM, Labella ML, Mullan RJ, Elamin MB, Erwin PJ \& Montori VM. Clinical review: insulin sensitizers for the treatment of hirsutism: a systematic review and metaanalyses of randomized controlled trials. Journal of Clinical Endocrinology and Metabolism 200893 1135-1142. (doi:10.1210/jc.2007-2429)

28 Kumar A, Woods KS, Bartolucci AA \& Azziz R. Prevalence of adrenal androgen excess in patients with the polycystic ovary syndrome (PCOS). Clinical Endocrinology 200562 644-649. (doi:10.1111/j.1365-2265.2005.02256.x)

29 Panidis D, Tziomalos K, Macut D, Delkos D, Betsas G, Misichronis G \& Katsikis I. Cross-sectional analysis of the effects of age on the hormonal, metabolic, and ultrasonographic features and the prevalence of the different phenotypes of polycystic ovary syndrome. Fertility and Sterility 201297 494-500. (doi:10.1016/ j.fertnstert.2011.11.041)

30 Carmina E, Rosato F, Jannì A, Rizzo M \& Longo RA. Extensive clinical experience: relative prevalence of different androgen excess disorders in 950 women referred because of clinical hyperandrogenism. Journal of Clinical Endocrinology and Metabolism 200691 2-6. (doi:10.1210/jc.2005-1457) 
31 Dilbaz B, Ozkaya E, Cinar M, Cakir E \& Dilbaz S. Cardiovascular disease risk characteristics of the main polycystic ovary syndrome phenotypes. Endocrine 201139 272-277. (doi:10.1007/s12020-011-9437-6)

32 Welt CK, Gudmundsson JA, Arason G, Adams J, Palsdottir H, Gudlaugsdottir G, Ingadottir G \& Crowley WF. Characterizing discrete subsets of polycystic ovary syndrome as defined by the Rotterdam criteria: the impact of weight on phenotype and metabolic features. Journal of Clinical Endocrinology and Metabolism 200691 4842-4848. (doi:10.1210/jc.2006-1327)

33 Guastella E, Longo RA \& Carmina E. Clinical and endocrine characteristics of the main polycystic ovary syndrome phenotypes. Fertility and Sterility 201094 2197-2201. (doi:10.1016/ j.fertnstert.2010.02.014)
34 Hahn S, Bering van Halteren W, Roesler S, Schmidt M, Kimmig R, Tan S, Mann K \& Janssen OE. The combination of increased ovarian volume and follicle number is associated with more severe hyperandrogenism in German women with polycystic ovary syndrome. Experimental and Clinical Endocrinology $\mathcal{E}$ Diabetes 2006114 175-181. (doi:10.1055/s-2006-924063)

Received 13 January 2013

Revised version received 11 March 2013

Accepted 4 April 2013 